\title{
Synthesis, chemical characterization, and $\mu$-opioid receptor activity assessment of the emerging group of 'nitazene' 2-benzylbenzimidazole synthetic opioids
}

\author{
Marthe Vandeputte ${ }^{1}$, Katleen Van Uytfanghe ${ }^{1}$, Nathan Layle ${ }^{2}$, Danielle St. Germaine ${ }^{2}$, \\ Donna Iula ${ }^{2}$, and Christophe Stove ${ }^{1}$ \\ ${ }^{1}$ Ghent University Faculty of Pharmaceutical Sciences \\ ${ }^{2}$ Cayman Chemical Company
}

March 15, 2021

\begin{abstract}
Several 2-benzylbenzimidazole opioids (also referred to as 'nitazenes') recently emerged on the illicit market. The most frequently encountered member, isotonitazene, has been identified in multiple fatalities since its appearance in 2019. Although recent scheduling efforts targeted isotonitazene, many other analogues remain unregulated. Being structurally unrelated to fentanyl, little is known about the harm potential of these compounds. In this study, ten nitazenes and four metabolites were synthesized, analytically characterized via four different techniques, and pharmacologically evaluated using two cell-based $\beta$-arrestin $2 /$ miniGi recruitment assays monitoring $\mu$-opioid receptor (MOR) activation. Based on absorption spectra and retention times, high-performance liquid chromatography coupled to diode-array detection (HPLC-DAD) allowed differentiation between most analogues. Time-of-flight mass spectrometry (LC-QTOF-MS) identified a fragment with $\mathrm{m} / z$ 100.11 for 12/14 compounds, which could serve as a basis for MS-based nitazene screening. MOR activity determination confirmed that nitazenes are generally highly active, with potencies and efficacies of several analogues exceeding that of fentanyl. Particularly relevant is the unexpected very high potency of the $N$-desethyl-isotonitazene metabolite, rivalling the potency of etonitazene and exceeding that of isotonitazene itself. Supported by its identification in fatalities, this likely has in vivo consequences. These results improve our understanding of this emerging group of opioids by laying out an analytical framework for their detection, as well as providing important new insights into their MOR activation potential.
\end{abstract}

